\title{
Efectividad del Plasma Rico en Plaquetas en la Cicatri- zación de Implantes Dentales: Una Revisión Sistemática
}

\author{
Effectiveness of Platelet Rich Plasma in the Healing of Dental Implants: \\ A Systematic Review
}

Eduardo Bravo S. \& Patricio Oliva M.

BRAVO, S. E. \& OLIVA, M. P. Efectividad del plasma rico en plaquetas en la cicatrización de implantes dentales: una revisión sistemática. Int. J. Odontostomat., 7(1):87-92, 2013.

RESUMEN: El plasma rico en plaquetas es un producto de la centrifugación diferencial de la sangre, logrando una alta concentración de plaquetas (600.000 a $1.500 .000 \times \mathrm{mm} 3)$, que al combinarse con cloruro de calcio comienza su activación, produciendo regeneración celular. Se ocupa actualmente dentro de procedimientos estéticos y quirúrgicos. En implantes dentales posee una efectividad por sobre el $92 \%$, además disminuye el tiempo para la rehabilitación, reduciendo la reabsorción ósea luego de la exodoncia. El objetivo del presente estudio fue evaluar el efecto del tratamiento con PRP en cirugías de implantes dentales. Se realizó una revisión sistemática cuya metodología consistió en revisar ensayos clínicos aleatorizados del tema. Se buscó en PUBMED, Lylacs, Blackwell y Ebsco, en la biblioteca Cochrane y en literatura gris mediante búsqueda manual. Se consideró los siguientes criterios de selección: mayores de 18 años, candidatos a tratamientos rehabilitadores con implantes dentales, sin alteraciones sistémicas de importancia. La estrategia se resume en los siguientes puntos: Identificación del problema, Identificación de términos de búsqueda, Búsqueda sistemática, Selección de artículos mediante utilización de guías de análisis crítico, Incorporación de la información al REVMAN, Análisis de sensibilidad de los artículos y Meta análisis. Se concluye que la mezcla del plasma rico en plaquetas puede inducir una reacción más fuerte de hueso alrededor del implante

PALABRAS CLAVE: plasma rico en plaquetas, implantes dentales, cicatrización.

\section{INTRODUCCIÓN}

El plasma rico en plaquetas es un adhesivo de fibrina autóloga con una alta concentración de plaquetas obtenido de la sangre por centrifugación (Antoniades, 1981; Anitua, 1999). Con factores de crecimientos (tanto mitogénicos como angiogénicos) implicados en la reparación y cicatrización del hueso, como factor de crecimiento transformante (TGF), derivado del factor de crecimiento plaquetario (PDGF) y muy similares al factor de crecimiento de la insulina (Giannobile et al., 1996).

Como el PRP es desarrollado por la sangre de cada paciente, por lo tanto es biocompatible y libre de enfermedades transmisibles, además posee un costo inferior en comparación a los factores de crecimiento recombinantes. Entonces, el efecto positivo del plasma rico en plaquetas puede ser atribuido a la angiogenética, específicamente a los procesos de proliferación y diferenciación en osteoblastos de los factores de crecimiento factor-b (TGF-b) y del factor de crecimiento plaquetario (PDGF) que están en una alta concentración en este tipo de plasma (Anitua).

En odontología se ha utilizado para la regeneración de tejidos posterior a alguna intervención (Blus et al., 2008), incluyendo tejido blando y hueso (Consolo et al., 2007), con resultados clínicamente significativos, aunque en ciertos tipos de cirugías su uso no está tan evidenciado (Kfir et al., 2007) o que simplemente demuestran resultados contradictorios o negativos en lo que ha cicatrización se refiere (Torres et al., 2009) lo que permite establecer la discusión sobre la real efectividad de este procedimiento e contextos clínicos. 
Por lo cual, el objetivo de este estudio radica en evaluar la efectividad del plasma rico en plaquetas en la cicatrización de implantes dentales mediante un procedimiento de revisión sistemática que deriva en un metaanálisis permitiendo generar una recomendación clínica basada en la evidencia.

\section{MATERIAL Y MÉTODO}

Se realizó una revisión sistemática donde fueron analizados ensayos clínicos. Los estudios evaluados están relacionados con el PRP y con la cirugía de implantes dentales, tanto en mandíbulas con problemas estructurales, como también en maxilares con elevación de seno maxilar e injertos óseos para darle mayor altura o estabilidad.

La investigación se articula mediante la siguiente pregunta de búsqueda: ¿Es efectivo el uso del plasma rico en plaquetas en la cicatrización de implantes dentales?

\section{Extracción de Datos}

Criterios de inclusión: Los registros recolectados correspondieron a información perteneciente ensayos clínicos randomizados que incorporarán: pacientes sometidos a cirugías maxilofaciales, con rehabilitación protésica, pacientes con implantes dentales, pacientes con tratamientos orales que utilicen plasma rico en plaquetas como coadyuvante de la cicatrización. Además, de artículos en idioma español, inglés, portugués.

Criterios de exclusión: Se excluyeron los artículos que presentaban la información de forma confusa y aquellos estudios mal indexados.

Estrategia de Búsqueda Se buscó en las bases de datos tales como Pubmed, Lylacs, Blackwell, y Ebsco, además de buscar en bibliotecas de universidades. Se contactó a los investigadores principales de cada investigación en situaciones particulares con el objeto de aclarar coneptos o solicitar información adicional. Se realizó con dos revisores independientes (Fig. 1).

Para la búsqueda se utilizaron las siguientes palabras claves: plasma rich plaquets, implants, dental implants, cicatrization implants, prp and implant, con los siguientes términos booleanos: AND y OR para encontrar relaciones entre los implantes dentales y los injertos óseos junto a rehabilitaciones posteriores.

Para la intervención, se utilizaron artículos en los cuales se evaluó la mejora de la capacidad rehabilitadora de los implantes. Se excluyeron estu-

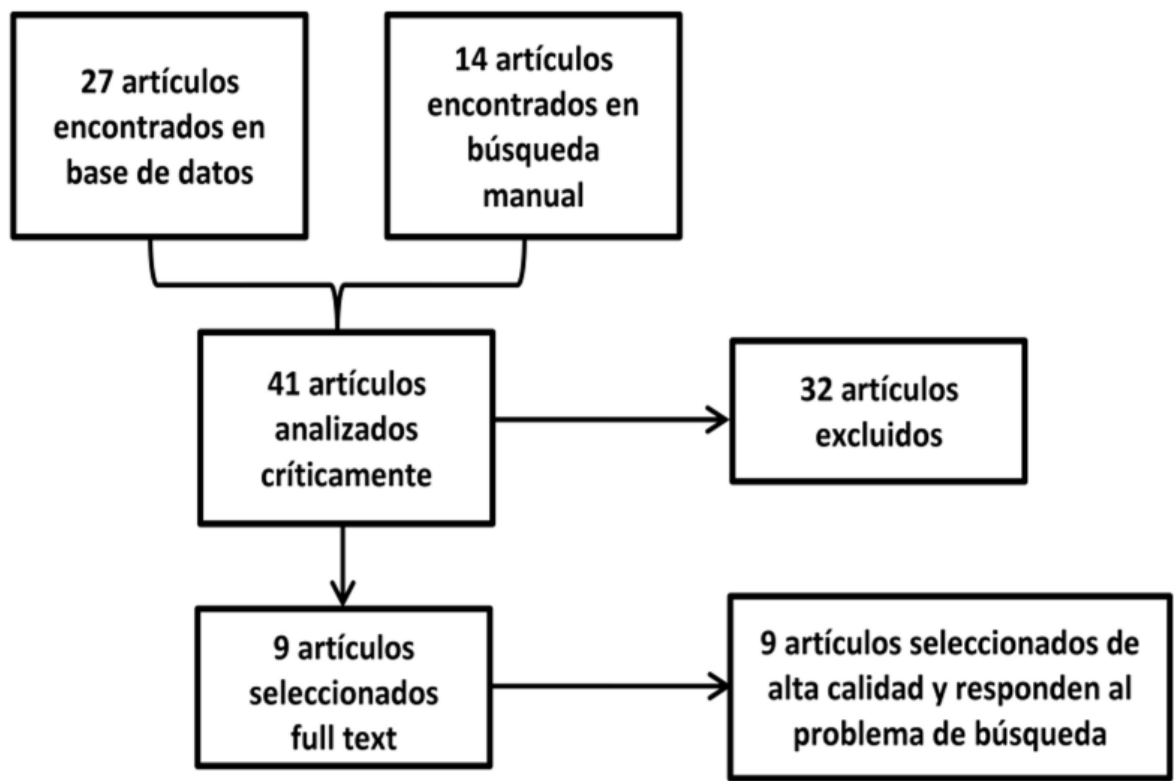

Fig. 1. Estructura de la Búsqueda Sistemática. 
dios que no cumplían con los criterios de selección o que se enfocaban indirectamente con el objeto de estudio

Se recopilaron 41 artículos que respondían al concepto de plasma rico en plaquetas como solución o coadyuvante a la rehabilitación sobre implantes en 14 se consiguió mediante búsqueda manual.

Se aplicó la guía Caspe de evaluación de ensayos clínicos para el análisis crítico de cada artículo, evaluada la calidad metodológica de éstos, aquellos que fueron seleccionados se ingresaron al programa Review Manager para el meta análisis final.

Análisis estadístico. Se evaluó mediante un modelo de efecto fijo, considerando que la variabilidad de los estudios es producida por el azar. Se utiliza Mantel Haenszel para el análisis de resultados ya que soporta estudios con muestras pequeñas y escasos eventos, como ocurre en la mayoría de los estudios seleccionados.

La heterogeneidad se evalúa mediante el $Q$ de Cochran con un $\mathrm{x} 2$, (que mide la presencia de heterogeneidad) además del I2, que expresa el porcentaje de variabilidad de resultados en los estudios.

\section{RESULTADOS}

El número de estudios que cumplían con los criterios de inclusión (previamente analizados) fueron 9 artículos científicos (Tabla I), sumando una cantidad de 1556 pacientes de forma global. Para el análisis se opta por el modelo de efecto fijo, asumiendo que cualquier variación observada en las puntuaciones se debió al error experimental de cada estudio particular, todo con una confiabilidad de un $95 \%$.

De los estudios seleccionados se efectúa el metaanálisis (Fig. 2), del cual se desprenden los procesos analíticos que en su conjunto derivan en la evaluación de la heterogeneidad de los resultados, permitiendo la recomendación de procedimiento.

El metaanálisis general presenta una heterogeneidad (I2) del $82 \%$, considerada como alta, superando el $75 \%$ (Higgins et al., 2003) que se establece como límite superior ve variabilidad de resultados de los estudios; con una significatividad del 0,00 (menor a 0,05) en la prueba de $\times 2$, que demuestra la presencia de heterogeneidad (concordando con el resultado del I2), lo cual implica una amplia dispersión de los resultados de los estudios particulares, especialmente por

\begin{tabular}{|c|c|c|c|c|c|c|c|c|}
\hline Study or Subgroup & \multicolumn{2}{|c|}{ PRP } & Control & $\begin{array}{l}\text { ol } \\
\text { Total }\end{array}$ & \multicolumn{2}{|r|}{ Odds Ratio } & \multicolumn{2}{|c|}{$\begin{array}{c}\text { Odds Ratio } \\
\text { M-H, Fixed, } 95 \% \mathrm{Cl}\end{array}$} \\
\hline Anitua 2008 & 197 & 220 & 837 & 840 & $38.9 \%$ & $0.03[0.01,0.10]$ & 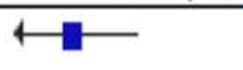 & \\
\hline Blus 2008 & 41 & 41 & 10 & 12 & $0.2 \%$ & $19.76[0.88,443.50]$ & & \\
\hline Caubet 2011 & 9 & 34 & 31 & 34 & $24.4 \%$ & $0.03[0.01,0.14]$ & $\hookleftarrow$ & \\
\hline Efrain Kfir $200 ?$ & 3 & 8 & 5 & 7 & $3.6 \%$ & $0.24[0.03,2.12]$ & & \\
\hline Filho Cerruti $200 ?$ & 30 & 32 & 30 & 32 & $2.0 \%$ & $1.00[0.13,7.57]$ & & \\
\hline Lee 2009 & 37 & 37 & 36 & 39 & $0.5 \%$ & $7.19[0.36,144.17]$ & & \\
\hline Rabelo 2010 & 11 & 92 & 17 & 44 & $21.7 \%$ & $0.22[0.09,0.52]$ & & \\
\hline Schaaf 2007 & 10 & 34 & 7 & 19 & $6.8 \%$ & $0.71[0.22,2.34]$ & & \\
\hline Torres Tamini 2007 & 151 & 153 & 124 & 129 & $1.9 \%$ & $3.04[0.58,15.96]$ & & \\
\hline Total (95\% Cl) & & 651 & & 1156 & $100.0 \%$ & $0.28[0.19,0.41]$ & & \\
\hline Total events & 489 & & 1097 & & & & & \\
\hline $\begin{array}{l}\text { Heterogeneity. } \mathrm{Chi}^{2}= \\
\text { Test for overall effect: }\end{array}$ & $\begin{array}{l}008, \mathrm{df}= \\
=6.28(\mathrm{f}\end{array}$ & $\begin{array}{l}=8(\mathrm{P}< \\
0<000\end{array}$ & $\begin{array}{l}0.00001 \\
0001)\end{array}$ & ; & & & \begin{tabular}{|lll}
0.01 & 0.1 & \\
& & \\
& PRP
\end{tabular} & $\begin{array}{l}10 \\
\text { CONTROL }\end{array}$ \\
\hline
\end{tabular}

Fig. 2. Metaanálisis de PRP. 
Tabla I. Estudios Incluidos.

\begin{tabular}{lll}
\hline Estudio incluido & \multicolumn{1}{c}{ Intervención } & \multicolumn{1}{c}{ Outcome } \\
\hline $\begin{array}{l}\text { Sinus graft with safescraper: } \\
\text { results. Caubet et al. (2011). }\end{array}$ & Estudio experimental & $\begin{array}{l}\text { En controles posteriores se vio radiográficamente que el } \\
\text { hueso se regenero en un 100\% y los implantes permanecieron } \\
\text { en su posición sin complicaciones posteriores. }\end{array}$
\end{tabular}

Effect of platelet-rich plasma on sinus lifting: a randomized-controlled clinical trial. Torres et al. (2009).

5-year clinical experience with BTI dental implants: risk factors for implant failure. Anitua et al. (2008)

Immediate loading of the grafted maxillary sinus using platelet rich plasma and autogenous bone: a preliminary study with histologic and histomorphometric analysis.v Lee et al. (2009)

Sinus bone grafting procedures using ultrasonic bone surgery: 5-year experience. Blus et al. (2008).
Topical use of platelet-rich plasma to influence bone volume in maxillary augmentation: a prospective randomized trial. Schaaf et al. (2008).

Allogenous bone grafts improved by bone marrow stem cells and platelet growth factors: clinical case reports. Filho Cerruti et al. (2007).
Ensayo prospectivo aleatorizado

Reportes de casos clínicos, 32 pacientes

15 casos de implantes consecutivos.

Immediate bone augmentation after infected tooth extraction using titanium membranes. Kfir et al. (2007).

Retrospective study of bone grafting procedures before implant placement. Rabelo et al. (2010). un periodo de 7 años
Ensayo clínico aleatorizado

Estudio experimental, seguimiento clínico a un años

Estudio retrospectivo y análisis histomorfométrico

Estudio retrospectivo para analizar el uso de cirugía piezoeléctrica por 5 años. grupo de pacientes por 5

PRP no es un factor determinante en la mejora de cicatrización junto a elevación de seno, pero si muestra una mejora en la conductividad del hueso, generando un mayor volumen de hueso formado.

El hábito de fumar, la posición del implante, la puesta en escena de implantes (los implantes de dos fases) y la aplicación de técnicas especiales fueron estadísticamente correlacionada con una menor supervivencia del implante.

Los resultados preliminares de este estudio retrospectivo sugieren que en implantes de carga inmediata para el seno maxilar injertado presenta altas tasas de supervivencia, y se puede lograr en un corto periodo de tiempo de curación en comparación con los convencionales protocolos Branemark, cuando se utiliza una proporción de 50:50 compuesto de FHA y hueso autógeno con PRP

El resultado una tasa de perforación de la membrana de 3,8\%, que fue baja en comparación con otros estudios (media $28,6 \%$ ). Esto se asocia típicamente con la presencia de un tabique con PRP dando lugar a u na mejor cirugía relativamente libre de la sangre, mejorar la vi sibilidad y la morbilidad menos tejido blando en las zonas donantes. Se tomó nota para mejorar el acceso y l a eficiencia del corte sobre el Mectron.

La histomorfometría no muestra mejores sustanciales en ningún tratamiento para la obt ención de un mayor volumen óseo.

La gran ventaja de la utilización de la fracción multinacional compuesta por el MSC y precursores hematopoyéticos y factores de crecimiento (PRP) es que promueve la cicatrización adecuada y la integración del injerto. Nuestros datos sugieren que en el futuro podría dirigir la investigación hacia la obtención de una expansión in vitro de las células nucleadas en hueso autólogo, y posiblemente a infundir en el paciente una mayor concentración.

Se necesitan más estudios investigar si las membranas de titanio son más resistente a la acumulación de placa y la infección de otros materiales de la membrana sometida a principios de la membrana la exposición

Estudio retrospectivo en La reconstrucción alveolar usando un hueso autógeno seguido por la colocación del implante es un tratamiento confiable para los pacientes con hueso insuficiente aquellos que se orientan hacia el control (Lee et al., 2009; Blus et al.; Torres et al.) que además poseen un bajo peso (Blus et al., 0,2\%; Lee et al., 0,5\%; Torres et al., 1,9\%); muestras bajas e intervalos de confianza amplios (Blus et al., 0,8-443; Lee et al., 0,3-144; Torres et al., 0,58-15,9).
La medida de resumen se sitúa en el Grupo de PRP, estableciendo la recomendación clínica de su uso.

Análisis de Sensibilidad. Para el análisis de sensibilidad se opta por restan los estudios que poseen un peso bajo (bajo $2 \%$ ) y cuyos intervalos de confianza son 
PRP

\begin{tabular}{|c|c|c|c|c|c|}
\hline \multirow[b]{2}{*}{ Study or Subgroup } & \multicolumn{2}{|c|}{ PRP } & \multicolumn{2}{|c|}{ Control } & \multirow[b]{2}{*}{ Weight } \\
\hline & Events & Total & Events & Total & \\
\hline Anitua 2008 & 197 & 220 & 837 & 840 & $40.8 \%$ \\
\hline Blus 2008 & 41 & 41 & 10 & 12 & $0.0 \%$ \\
\hline Caubet 2011 & 9 & 34 & 31 & 34 & $25.6 \%$ \\
\hline Efrain Kfir 2007 & 3 & 8 & 5 & 7 & $3.7 \%$ \\
\hline Filho Cerruti 2007 & 30 & 32 & 30 & 32 & $0.0 \%$ \\
\hline Lee 2009 & 37 & 37 & 36 & 39 & $0.0 \%$ \\
\hline Rabelo 2010 & 11 & 92 & 17 & 44 & $22.7 \%$ \\
\hline Schaaf 2007 & 10 & 34 & 7 & 19 & $7.1 \%$ \\
\hline Torres Tamini 2007 & 151 & 153 & 124 & 129 & $0.0 \%$ \\
\hline Total $(95 \% \mathrm{Cl})$ & & 388 & & 944 & $100.0 \%$ \\
\hline Total events & 230 & & 897 & & \\
\hline $\begin{array}{l}\text { Heterogeneity: } \mathrm{Chi}^{2} \\
\text { Test for overall effec }\end{array}$ & $\begin{array}{l}37, \mathrm{df} \\
=8.08\end{array}$ & & $\begin{array}{l}0.001) ; \\
001)\end{array}$ & $=789$ & \\
\hline
\end{tabular}

Odds Ratio Odds Ratio

$\mathrm{M}-\mathrm{H}$, Fixed, $95 \% \mathrm{Cl} \quad \mathrm{M}-\mathrm{H}$, Fixed, $95 \% \mathrm{Cl}$

Fig. 3. Evaluación de Sensibilidad.

amplios, se expresan variaciones evidentes en los resultados de metaanálisis (Fig. 3), con un OR final de 0,13 con intervalos de 0,08 y 0,21 , y una heterogeneidad (I2) que también disminuye al $78 \%$ (acercándose al límite medio de un $75 \%$ ).

La medida de resumen se mantiene en el PRP, por lo cual se asume que aumenta con ello la efectividad de la terapia por sobre otras para la cicatrización. La medida de resumen se sitúa en el Plasma Rica en Plaqueta, lo que concuerda con las medidas anteriormente señaladas.

Seguridad del tratamiento. Los estudios no evidencian efectos secundarios en el proceso de la aplicación de la intervención del Plasma Rico en Plaquetas. Las complicaciones anunciadas en los artículos se asocian con las cirugías de implantes más que con el procedimiento en sí.

\section{DISCUSIÓN}

El propósito del estudio radica en la evaluación de la efectividad del uso del plasma rico en plaquetas en cirugías de implantes dentales. De acuerdo a la evidencia se demuestra que el efecto local del plasma rico en plaquetas actúa en la cicatrización temprana de hueso cortical en tejido perimplantario (Nikoladikis et al., 2008), mejorando las propiedades osteoconduc-tivas de los injertos óseos, aumentando el volumen de hue- so nuevo formado (Torres et al.; Lee et al.), ratificado por seguimientos radiográficos e histomor-fométricos, que mostraron mayores valores de densitometría en las zonas de elevación de seno con injerto (Consolo et al.), manteniendo y aumentando sus propiedad en la combinación de fibrina-plasma rico en plaquetas enriquecido. Si bien existen resultados que demuestran el efecto contrario, gran parte de la población estudiada presenta un efecto positivo ante el tratamiento de pasma rico en plaquetas para la intervención señalada.

Los estudios (realizado el análisis de sensibilidad) señalan la efectividad de este procedimiento en el proceso de cicatrización en cirugías de implantes dentales, a lo que se suma una baja contraindicación debido a que existen pocos efectos adversos que se puedan asociar al procedimiento (sobre todo considerando el tema de la biocompatibilidad), resultando apropiado incluso para la regeneración de hueso en tratamientos cortos (Consolo et al.).

Recomendaciones. El plasma rico en plaquetas presenta una alta concentración de factores de crecimiento y de plaquetas, mostrando en este análisis que al combinarlo con rehabilitaciones mediante implantes dentales, con cirugía de elevaciones de seno y de injertos óseos, se obtiene una mejora en calidad de hueso y en el tipo de cicatrización, por lo cual se evidencia que aumenta con ello la efectividad de la terapia en sí, por sobre otras alternativas para la cicatrización y posterior rehabilitación, mejorando el tratamiento, logrando una mayor estabilidad y calidad de cicatrización. 
BRAVO, S. E. \& OLIVA, M. P. Effectiveness of platelet rich plasma in the healing of dental implants: a systematic review. Int. J. Odontostomat., 7(1):87-92, 2013.

ABSTRACT: The platelet rich plasma is a differential centrifugation product of the blood, getting a high concentration of platelets $(600.000$ to $1.500 .000 \times \mathrm{mm} 3)$, which when combined with calcium chloride begins its activation, resulting in cell regeneration. It is currently in aesthetic and surgical procedures. Dental implant has effectiveness over $92 \%$, and decreases the time for rehabilitation, reducing bone resorption after extraction. The objective of this study was to evaluate the effect of treatment with PRP in dental implant surgery. We conducted a systematic review whose methodology was to review randomized clinical trials of the subject. We searched PUBMED, Lylacs, Blackwell and Ebsco, the Cochrane Library and books. We considered the following selection's criteria: over 18 years, candidates for dental implant rehabilitation treatment, without significant systemic changes. The strategy is summarized in the following: Identify the problem, identification of search terms, systematic search, selection of articles by using critical thinking guides, Incorporation of information to REVMAN, Sensitivity analysis of the articles, analysis of bias and Meta analysis. We conclude that the mixture of platelet affluent plasma can induce a stronger reaction of bone around the implant

KEY WORDS: platelet rich plasma, dental implants, scarring.

\section{REFERENCIAS BIBLIOGRÁFICAS}

Anitua, E. Plasma rich in growth factors: preliminary results of use in the preparation of future sites for implants. Int. J. Oral Maxillofac. Implants, 14(4):529-35, 1999.

Anitua, E.; Orive, G.; Aguirre, J. J.; Ardanza, B. \& Andía, I. 5year clinical experience with BTI dental implants: risk factors for implant failure. J. Clin. Periodontol., 35(8):72432,2008

Antoniades, H. N. Human platelet-derived growth factor (PDGF): purification of PDGF-I and PDGF-II and separation of their reduced subunits. Proc. Natl. Acad. Sci. U S A, 78(12):7314-7, 1981

Blus, C.; Szmukler-Moncler, S.; Salama, M.; Salama, H. \& Garber, D. Sinus bone grafting procedures using ultrasonic bone surgery: 5-year experience. Int. J. Periodontics Restorative Dent., 28(3):221-9, 2008.

Caubet, J.; Petzold, C.; Sáez-Torres, C.; Morey, M.; Iriarte, J. I.; Sánchez, J., et al. Sinus graft with safescraper: 5-year results. J. Oral Maxillofac. Surg., 69(2):482-90, 2011.

Consolo, U.; Zaffe, D.; Bertoldi, C. \& Ceccherelli, G. Plateletrich plasma activity on maxillary sinus floor augmentation by autologous bone. Clin. Oral Implants Res., 18(2):25262, 2007.

Filho Cerruti. H.; Kerkis, I.; Kerkis, A.; Tatsui, N. H.; da Costa Neves, A.; Bueno, D. F.; et al. Allogenous bone grafts improved by bone marrow stem cells and platelet growth factors: clinical case reports. Artif. Organs, 31(4):268-73, 2007.

Giannobile, W. V.; Hernandez, R. A.; Finkelman, R. D.; Ryan, S.; Kiritsy, C. P.; D'Andrea, M.; et al. Comparative effects of platelet-derived growth factor-BB and insulin-like growth factor-I, individually and in combination, on periodontal regeneration in Macaca fascicularis. J. Periodontal Res., 31(5):301-12, 1996.
Higgins, J. P.; Thompson, S. G.; Deeks, J. J. \& Altman, D. G. Measuring inconsistency in meta-analyses. BMJ, 327(7414):557-60, 2003.

Kfir, E.; Kfir, V. \& Kaluski, E. Immediate bone augmentation after infected tooth extraction using titanium membranes. J. Oral Implantol., 33(3):133-8, 2007.

Lee, C. Y.; Rohrer, M. D.; Prasad, H. S.; Stover, J. D. \& Suzuki, J. B. Sinus grafting with a natural fluorohydroxyapatite for immediate load: a study with histologic analysis and histomorphometry. J. Oral Implantol., 35(4):164-75, 2009.

Nikolidakis, D.; van den Dolder, J.; Wolke, J. G. \& Jansen, J. A. Effect of platelet-rich plasma on the early bone formation around $\mathrm{Ca}-\mathrm{P}$-coated and non-coated oral implants in cortical bone. Clin. Oral Implants Res., 19(2):207-13, 2008.

Rabelo, G. D.; de Paula, P. M.; Rocha, F. S.; Jordão Silva, C. \& Zanetta-Barbosa, D. Retrospective study of bone grafting procedures before implant placement. Implant Dent., 19(4):342-50, 2010.

Schaaf, H.; Streckbein, P.; Lendeckel, S.; Heidinger, K.; Görtz, B.; Bein, G.; et al. Topical use of platelet-rich plasma to influence bone volume in maxillary augmentation: a prospective randomized trial. Vox Sang., 94(1):64-9, 2008.

Torres, J.; Tamimi, F.; Martinez, P. P.; Alkhraisat, M. H.; Linares, R.; Hernández, G.; et al. Effect of platelet-rich plasma on sinus lifting: a randomized-controlled clinical trial. J. Clin. Periodontol., 36(8):677-87, 2009.

Dirección para correspondencia:

Eduardo Bravo S.

Facultad de Odontología

Universidad del Desarrollo

Barros Arana 1734

Concepción - CHILE

Email: edu.bravo.s85@gmail.com Recibido: 21-06-2012 Aceptado: 22-10-2012 